\title{
Multi-model Predictive Stability Control Strategy for Vehicle Four-wheel Steering System Considering Tire Nonlinear Cornering Characteristics
}

\section{Zhongkai Luan}

Nanjing University of Aeronautics and Astronautics

Wanzhong Zhao (D zhaowanzhong@126.com)

Nanjing University of Aeronautics and Astronautics

Chunyan Wang

Nanjing University of Aeronautics and Astronautics

\section{Research Article}

Keywords: Four-wheel steering system, Deep Learning Framework, Tire Nonlinear Cornering Characteristics, LSTM, Model Predictive Control, Phase Plane Method

Posted Date: March 7th, 2022

DOI: https://doi.org/10.21203/rs.3.rs-1407088/v1

License: (c) (1) This work is licensed under a Creative Commons Attribution 4.0 International License. Read Full License 


\section{Multi-model Predictive Stability Control Strategy for Vehicle Four-wheel Steering System Considering Tire Nonlinear Cornering Characteristics}

\section{Zhongkai Luan, Wanzhong Zhao*, Chunyan Wang}

\begin{abstract}
In extreme working conditions such as poor road conditions, the low road adhesion coefficient is easy to cause the tire cornering characteristics curve to be in the nonlinear domain, making the vehicle system in the critical instability state. To this end, this paper proposes a cascade deep learning framework combining multi-model predictive control (MMPC) and LSTM tire cornering stiffness estimation (TCSE) neural network and designs a stability control strategy of vehicle four-wheel steering system considering tire nonlinear cornering characteristics. The four-wheel steering system and vehicle tire dynamic model are analyzed and established, and the online estimation method of tire cornering stiffness and MMPC's sub-model classification method is developed. On this basis, the tire angle is creatively introduced as the phase plane stability region boundary, used to design the MMPC controller's boundary condition. The hardware in the loop test results shows that compared with the existing research, the strategy proposed in this paper can effectively improve the tracking accuracy of the target steering signal and ensure the system's stability when the road adhesion coefficient is low.
\end{abstract}

Keywords: Four-wheel steering system, Deep Learning Framework, Tire Nonlinear Cornering Characteristics, LSTM, Model Predictive Control, Phase Plane Method

\section{Introduction}

In the steering process, the wheel's driving directional be separated from the wheel plane, and the tire sideslip will appear [1-3]. When the vehicle system is in the steering condition with a low road adhesion coefficient, it is easy to cause the tire cornering characteristics curve, that is, lateral force-sideslip angle curve, to be in the nonlinear domain or even the saturation domain, making the system close to the unstable state $[4,5]$. Compared with the front wheel steering system, the four-wheel steering system can change the

Zhongkai Luan, Wanzhong Zhao *, Chunyan Wang

College of Energy and Power Engineering, Nanjing University of Aeronautics and Astronautics

29 Yudao St.,Nanjing 210016,Jiangsu Province, People's Republic of China

Emali: zwz@nuaa.edu.cn response characteristics of lateral acceleration by adjusting the response characteristics of the rear-wheel angle [6, 7]. Therefore, when the cornering characteristics of tires show nonlinear characteristics, designing the stability control strategy of the four-wheel steering system considering tire cornering characteristics to minimize its adverse impact on the stability of the steering process is the critical factor in ensuring the stability of four-wheel steering system under various working conditions.

In recent years, the research on steering systems mainly focuses on steering stability under extreme conditions [8-10]. Taghavifara et al. employed a four-wheel active steering model and proposed a probabilistic estimation approach by hybridizing optimal robust control and a damped least-square backpropagation based neural networks (NN) to design a control system [11]. Chatzikomis et al. combined the electronic stability control system with the four-wheel steering system, indicating that the four-wheel steering system can continue to improve the driving safety of vehicles based on the electronic stability control system [12]. Hang et al. proposed a four-wheel independent steering system based on a steering system and designed a new sliding mode controller to suppress external interference [13]. However, the above research does not consider the nonlinear characteristics of the model caused by tire characteristics, sideslip angle, road adhesion coefficient, and other factors.

Given the above problems, Farroni et al. used numerical and graphical methods to show the physical characteristics in the form of saddle-node bifurcation parameters under the condition of considering the lateral load transfer borne by the tire and the interaction between the tire and the road state with the nonlinear magic formula [14]. From the above research, the state variables reflecting the system's stability are mainly yaw rate and sideslip angle. Among them, the yaw rate represents the change speed of heading angle during vehicle steering, and the sideslip angle describes the deviation degree of the motion trajectory direction of the vehicle system compared with the $\mathrm{x}$-axis of the body coordinate system. There is a coupling relationship between them [15-17]. 
Therefore, the phase plane analysis considering the sideslip angle can effectively express the influence of the sideslip angle on stability. The current research analyses the influence of the sideslip angle on the vehicle stability through the phase plane method, gives the criterion to judge the stability, and designs the critical parameters as the function related to the sideslip angle and the longitudinal speed of the vehicle, but ignores the influence of the wheel angle on the stability region. In addition, with the decrease of road adhesion coefficient, the corresponding tire sideslip angle will also decrease when the lateral force sideslip angle curve enters the nonlinear region, and the tire equivalent sideslip stiffness will change [18]. Model predictive control has strict requirements on the prediction model's accuracy, and the prediction model's error will affect the control effect [19, 20]. Designing the estimation strategy considering the calculation efficiency and accuracy is also critical in realizing system sub-model control when nonlinear tire cornering characteristics. Considering that the mapping of front and rear wheel cornering stiffness based on the dynamic model is time-dependent, that is, the vehicle dynamic state parameters can not be mutated, it is suitable to design the estimation strategy of tire equivalent cornering stiffness based on LSTM depth neural network [21, 22]. However, the existing research rarely considers the model parameter training with a small amount of data, which is limited by the difficulty of collecting a large amount of data. It will eventually affect the estimation accuracy of tire cornering stiffness.

To solve the above problems, this paper proposes a cascade deep learning architecture combining multi-model predictive control (MMPC) and adaptive learning rate LSTM neural network and designs a stability control strategy of vehicle four-wheel steering system considering tire nonlinear cornering characteristics. The four-wheel steering system and vehicle tire dynamic model are analyzed and established, and the online estimation method of tire cornering stiffness and MMPC's sub-model classification method is developed. On this basis, the tire angle is creatively introduced as the phase plane stability region boundary, used to design the MMPC controller's boundary condition. Compared with the existing research, the strategy proposed in this paper effectively realizes the stability control of the four-wheel steering system and ensures computational efficiency.

The contributions of this paper are as follows:

1) A cascade deep learning framework combining multi-model predictive control (MMPC) and tire cornering stiffness estimation (TCSE) neural network is proposed. In the rolling optimization process of MMPC, the controller switches the sub-model parameters in MMPC according to the current working domain of the tire output by the TCSE network. Compared with the existing research, this strategy balances the contradiction between computational efficiency and model accuracy.

2) An estimation strategy of tire cornering stiffness based on adaptive learning rate LSTM neural network is proposed. The attenuation rate of historical gradient value in the training process is controlled by introducing the attenuation rate parameter, which effectively solves the problem that the network falls into optimal local solution in the training process due to the small training data set.

3 ) Based on the phase plane method, the law between the sideslip angle of the four-wheel steering system and the range of the stability region is studied. The tire angle is creatively introduced as the boundary of the phase plane stability region of the four-wheel steering system under extreme conditions, which effectively realizes the stability control of the four-wheel steering system when the tire sideslip characteristics are nonlinear.

The remaining sections of this paper are arranged as follows: Section 2 is the stability control strategy architecture of the four-wheel steering system proposed in this paper. Section 3 establishes the dynamic model of the four-wheel steering system and its nonlinear vehicle-tire model. In Section 4, a multi-model predictive stability control strategy for the four-wheel steering system considering tire cornering characteristics is proposed. Section 5 is the results and discussion. Section 6 is the conclusion.

\section{Framework}

The stability control strategy of the four-wheel steering system considering tire nonlinear cornering characteristics and its hardware in the loop simulation system proposed in this paper is shown in Figure 1. Aiming at the problem that tire cornering characteristics will change dynamically with working conditions, this paper establishes the tire cornering stiffness estimation (TCSE) network shown in part a of Figure 1, which includes the estimation layer (EL) based on adaptive learning rate LSTM and the classification layer (CL). The output of the EL is the tire cornering stiffness of the front wheel and the rear wheel. According to the results of the EL, the region of tire cornering stiffness is divided into three categories: linear domain, nonlinear domain, and saturation domain.

Then, this paper establishes the multi-model predictive control (MMPC) strategy shown in part b of Figure 1, innovatively introduces the tire angle as the boundary of the phase plane stability region, and puts forward the MMPC 
stability strategy of the four-wheel steering system. The controller dynamically switches the sub-model parameters of the MMPC according to the output of the CL, which effectively balances the contradiction between the calculation efficiency and the model accuracy. In addition, to collect the data set used for TCSE network model parameter training and verify the strategy's effectiveness, this paper establishes hardware in the loop test platform as shown in part $\mathbf{c}$ of Figure 1. Based on

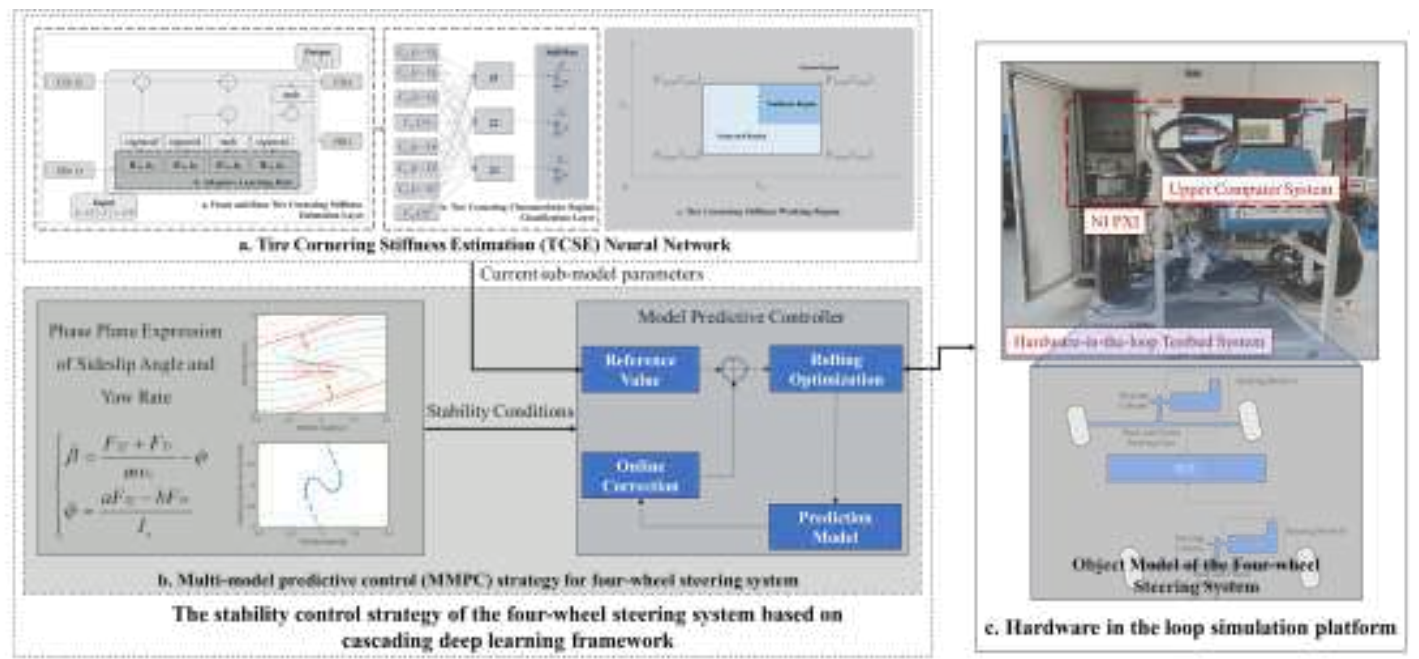

Fig. 1 The framework of stability control strategy of the four-wheel steering system and its hardware in the loop test system

CAN network communication, the steering system controller forms closed-loop data interaction with the virtual traffic environment in CarSim software through NI PXI.

\section{Dynamic Model of the Four-wheel Steering System}

In this section, through the analysis of the four-wheel steering system, the linear four-wheel steering system model and nonlinear vehicle-tire model are established, laying a foundation for the design of multi-model predictive stability control strategy for the four-wheel steering system considering the tire cornering characteristics.

\subsection{Linear four-wheel steering system model}

The dynamic model of the linear four-wheel steering system is analyzed and established. The four-wheel steering system includes two groups of front and rear steering mechanisms, which can reverse the phase of the front and rear wheels when steering at low speed, reduce the vehicle's steering radius, and improve the steering flexibility. Moreover, the front and rear wheels can steer in the same phase when steering at high speed to reduce the sideslip angle and improve the vehicle's handling stability [23]. Because the four-wheel steering system's front and rear steering mechanisms studied in this paper adopt the same structure, they are not distinguished in the modelling stage. The steering actuator, including the steering motor and steering gear, is modelled, as shown in Figure 2.

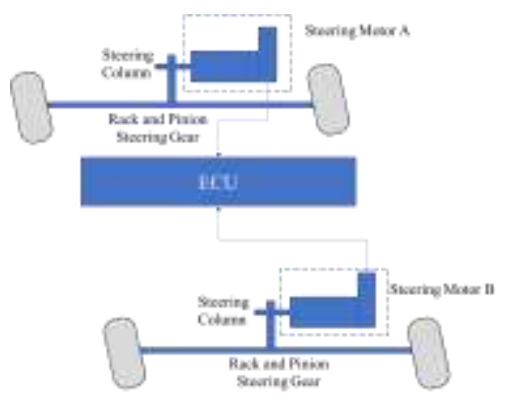

Fig. 2 Dynamic model of the four-wheel steering system

Firstly, the steering motor of the four-wheel steering system is analyzed, and the dynamic model is obtained, as shown in equation 1.

$$
\left\{\begin{array}{l}
L \&+R i+E_{m}=U \\
E_{m}=K_{b} \otimes_{m}^{\&}
\end{array}\right.
$$

where, $L$ is the armature inductance of the steering motor, $i$ is the armature current of the steering motor, $R$ is the armature resistance of the steering motor, $E_{m}$ is the back EMF of the steering motor, $U$ is the voltage at both ends of the armature of the steering motor, $K_{\mathrm{b}}$ is the back EMF constant of the steering motor, $\theta_{m}$ is the steering motor angle.

The four-wheel steering system studied in this paper adopts rack and pinion mechanism before and after steering, and its dynamic model is shown in equation 2 .

$$
\left\{\begin{array}{l}
M_{r}+B_{r} \underset{r}{\&}+F_{R}=N T_{a} / r_{p} \\
\theta_{s g}=x_{r} / r_{p}
\end{array}\right.
$$

where, $M_{r}$ is the mass of the rack, $B_{r}$ is the damping coefficient of the rack, $F_{R}$ is the steering resistance of the rack, $x_{r}$ is rack displacement, $\theta_{s g}$ is the pinion angle, $N$ is the 
reduction ratio of steering motor, $r_{p}$ is the pinion radius.

The rack force of the four-wheel steering system is equivalent to the steering column, and the dynamic model is shown in equation 3 .

$$
M_{r} \&_{s g}+B_{r} \Theta_{s g}^{\&}+T_{r}=N T_{a}
$$

where, $T_{r}$ is the tire return torque equivalent to the steering column.

\subsection{Nonlinear tire vehicle model}

The nonlinear tire model considering road adhesion coefficient is analyzed and established. Under the condition of low adhesion coefficient, the strong nonlinear characteristics faced by tires significantly impact vehicle stability control. Standard tire models include the linear tire, Pacejka magic formula, and Dugoff tire [24-26]. To consider the factors such as road adhesion coefficient and slip rate when analyzing the stress of tires, a nonlinear Dugoff tire model is established in this paper, as shown in Figure 3.

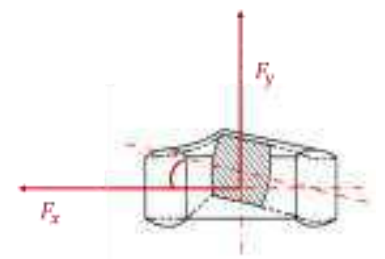

Fig. 3 Tire model

According to the definition of the Dugoff model, the longitudinal tire force and lateral force are related to the tire stiffness and related to the road adhesion coefficient and slip ratio [27]. The longitudinal force and lateral force of the Dugoff tire model are expressed as follows.

$$
\left\{\begin{array}{l}
F_{x}=C_{s} \frac{s}{1+s} f(\lambda) \\
F_{y}=C_{\alpha} \frac{\tan \alpha}{1+s} f(\lambda)
\end{array}\right.
$$

where, $F_{x}$ is the longitudinal force of the tire, $F_{y}$ is the tire lateral force, $C_{s}$ is the longitudinal stiffness of the tire, $C_{\alpha}$ is the tire cornering stiffness, $s$ is the longitudinal slip ratio of the tire, $\alpha$ is the sideslip angle of the tire. The expression of $f$ $(\lambda)$ is as follows:

$$
f(\lambda)=\left\{\begin{array}{cc}
(2-\lambda) \lambda & \lambda \leq 1 \\
1 & \lambda>1
\end{array}\right.
$$

where, $\lambda=\frac{\mu F_{z}(1+s)}{2 \sqrt{C_{s}^{2} s^{2}+C_{\alpha}^{2} \tan ^{2} \alpha}}, F_{z}$ is the vertical tire load.

Next, the nonlinear vehicle dynamics model is analyzed and established, as shown in equation 4 [28, 29]. Since the longitudinal speed is selected as the state quantity and the small-angle assumption is not made for the heading angle, the vehicle dynamics model is nonlinear and needs further processed in the subsequent controller design.

$$
\begin{aligned}
& y^{\&}=x \sin \varphi+\not \cos \varphi \\
& x^{\&}=\not \cos \varphi-\& \sin \varphi \\
& m \&=2 C_{\alpha f}\left(\delta_{f}-\frac{\&+a \phi \&}{\not \&}\right)+2 C_{\alpha r}\left(\delta_{r}-\frac{\& \& b \phi}{\& \&}\right)-m \& \& \\
& m 2 C_{s f} s_{f}+2 C_{s r} s_{r}+2 C_{\alpha f}\left(\delta_{f}-\frac{\& \&+a \phi \&}{\alpha}\right) \delta_{f}+2 C_{\alpha r}\left(\delta_{r}-\frac{\&-b \phi \&}{d \&}\right) \delta_{r} \\
& \phi \&=\phi \& \\
& I_{z}=2 a C_{\alpha f}\left(\delta_{f}-\frac{\&+a \phi \&}{\&}\right)-2 b C_{\alpha r}\left(\delta_{r}-\frac{\& \&-b c \&}{\&}\right)
\end{aligned}
$$

where, $Y$ is the lateral displacement of the global coordinate system, $X$ is the longitudinal displacement of the global coordinate system, $\&$ is the lateral velocity of the body coordinate system, $\&$ is the longitudinal velocity of the body coordinate system, $\varphi$ is the heading angle, $\varphi^{\&}$ is the yaw angle, $\delta_{f}$ is the front wheel angle, $\delta_{r}$ is the rear wheel angle, $a$ and $b$ are the distances from the centre of mass to the front and rear axles respectively, $I z$ is the moment of inertia of the system rotating around the $\mathrm{Z}$-axis.

\section{Multi-model Predictive Stability Control Strategy based on Cascading Deep Learning Framework}

Tire cornering stiffness is the ratio of tire lateral force to sideslip angle. Even for tires with the same structural parameters, the cornering stiffness value will change with working conditions, thus affecting the accuracy of vehicle stability control [30]. Based on the model established in Section 3, the tire cornering characteristics under different road adhesion coefficients are analyzed in Figure 4. It can be concluded that when the road adhesion coefficient decreases, the maximum value of tire lateral force will decrease, and the sideslip angle corresponding to the limit of tire lateral force will decrease. The nonlinear region of tire sideslip characteristics will increase with the decrease of road adhesion coefficient, resulting in the risk of sideslip of the four-wheel steering system. 


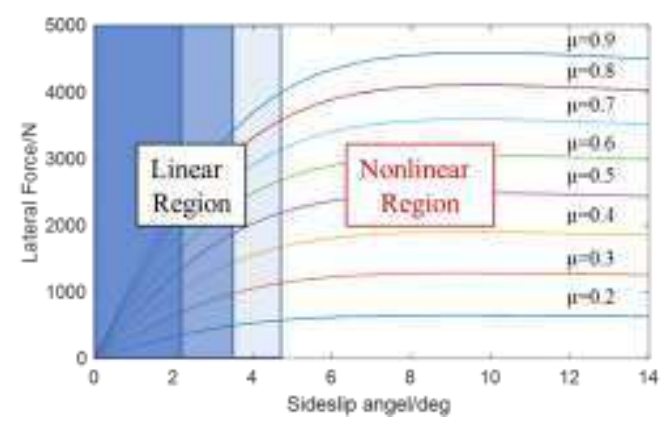

Fig. 4 Relationship between tire lateral force and sideslip angle under different road adhesion coefficients

To solve the above problems, this section proposes a cascade deep learning architecture combining multi-model predictive control (MMPC) and adaptive learning rate LSTM neural network and designs a stability control strategy of vehicle four-wheel steering system considering tire cornering characteristics.

4.1 Tire Cornering Stiffness Estimation (TCSE) Neural Network

This section proposes a tire cornering stiffness estimation (TCSE) neural network, including the estimation layer (EL) based on adaptive learning rate LSTM and the full connection classification layer (CL). Among them, the input of the EL is six measured signals, and the output is the tire cornering stiffness of the front and rear wheel. The input of the CL is the estimation result of the estimation layer. The output is the working domain of the tire cornering stiffness classified according to the current working conditions: linear domain, nonlinear domain, and saturation domain, respectively.

Firstly, the EL in the network model is analyzed. Through the analysis of the dynamic model of the four-wheel steering vehicle established in Section 3, it is obtained that there is a mapping relationship between the equivalent cornering stiffness and the state quantity of the four-wheel steering system, as shown in equation 7 .

$$
\left\{\begin{array}{l}
m a_{y}=C_{\alpha f} \alpha_{f}+C_{\alpha r} \alpha_{r}=C_{\alpha f}\left(\beta+\frac{a q \&}{v_{x}}-\delta_{f}\right)+C_{\alpha r}\left(\beta-\frac{b q \&}{v_{x}}-\delta_{r}\right) \\
I_{z} a C_{\alpha f} \alpha_{f}-b C_{\alpha r} \alpha_{r}=C_{\alpha f} a\left(\beta+\frac{a q \&}{v_{x}}-\delta_{f}\right)-C_{\alpha r} b\left(\beta-\frac{b q \&}{v_{x}}-\delta_{r}\right)
\end{array}\right.
$$

Analyze the four-wheel steering vehicle's dynamic model and select six signals: lateral acceleration, yaw angular acceleration, wheel angle, longitudinal speed, sideslip angle, and yaw rate as the input of the estimator. The input parameter data can be obtained through the inertial navigation system, as shown in equation 8 . In particular, the wheel angle is an array containing two sets of data: front wheel angle and rear wheel angle. The equivalent cornering stiffness of the front and rear wheel are selected as the estimator's output.

$$
\left\{\begin{array}{l}
\text { Input }:\left\{a_{y},\left[\delta_{f}, \delta_{r}\right], v_{x}, \beta, \alpha\right\} \\
\text { Output }:\left\{C_{\alpha f}, C_{\alpha r}\right\}
\end{array}\right.
$$

Considering that mapping the lateral stiffness of the front and rear wheels based on the dynamic model is time-dependent, the vehicle dynamic state parameters cannot be abrupt. Therefore, this paper designs an estimation strategy of tire equivalent cornering stiffness based on the LSTM neural network. In addition, considering that using the unified global learning rate, some parameters may have been optimized near the minimum. However, other parameters still have a significant gradient. This section proposes an estimation method of tire equivalent cornering stiffness based on adaptive learning rate LSTM neural network, as shown in Figure 5. The estimation method of tire equivalent cornering stiffness based on adaptive learning rate LSTM neural network shown in Figure 5 is discussed. Existing methods, such as AdaGrad, generally set different learning rates for each training parameter and automatically adapt to the learning of these parameters through some algorithms in the whole learning process [31]. However, the above algorithms need many data for training. Aiming at the problem of estimating the cornering stiffness of the front and rear wheels with small-batch training data, an adaptive learning rate algorithm RMSProp for the cornering stiffness of the front and rear wheels with small-batch training data is proposed in this paper. Compared with the fact that each parameter in AdaGrad is inversely proportional to the square root of the sum of the historical square values of all gradients, the algorithm proposed in this paper uses the exponential attenuation average method to weaken the influence of the historical value on the current value update. It introduces the attenuation rate parameter to control the attenuation rate of the historical gradient value. It can effectively solve the problem of network parameter training falling into optimal local solutions caused by insufficient training data set.

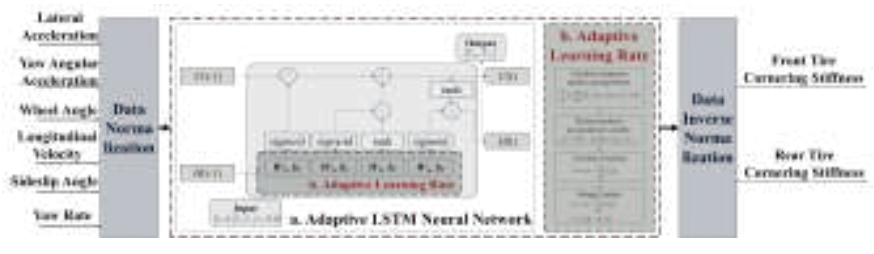

Fig. 5 Adaptive learning rate LSTM neural network tire equivalent cornering stiffness estimation method

The main body of the algorithm of adaptive learning rate is as follows:

1) Take out the small-batch data $\left\{a_{y}{ }^{i},\left[\delta_{f}{ }^{i}, \delta_{r}{ }^{i}\right], v_{x}{ }^{i}, \beta^{i}, \not \&\right\}$, and the target corresponding to 
the data is represented by $\left\{C_{\alpha f}{ }^{i}, C_{\alpha r}{ }^{i}\right\}$.

2) Calculate temporary updates.

$$
\omega+\varepsilon v \rightarrow \bar{\omega}
$$

where, $\omega$ is the initialization weight parameter, $\varepsilon$ is the momentum coefficient, $v$ is the initialization parameter.

Calculate the gradient based on small-batch data, as shown in equation 10 .

$$
\frac{1}{m} \forall_{\omega} \sum_{i} L\left[f\left(x_{i}, \omega\right), y_{i}\right] \rightarrow g
$$

(3) Accumulate the square gradient and refresh the gradient accumulation variable $r$, as shown in equation 11 .

$$
\rho r+(1-\rho) g \text { e } g \rightarrow r
$$

where, $\rho$ is the attenuation rate parameter of historical gradient value, $r$ is the gradient accumulation variable, and the initial value is 0 .

(4) Calculate gradient update.

$$
v=\varepsilon v-\frac{\alpha}{\sqrt{r}} \text { e } g
$$

(5) Update learning rate parameters according to $\Delta \omega$.

$$
v+\omega \rightarrow \omega
$$

Next, the LSTM neural network structure based on the adaptive learning rate is designed. It includes two main parameters: long memory $\mathrm{c}$ and short memory $\mathrm{h}$, and three main structures: input gate, output gate, and forgetting gate[32-34]. The equations of each part are deduced below.

Determine the historical information be forgotten based on the sigmoid function. After $f_{t}$ is obtained, it is multiplied by the long memory parameter $\mathrm{C}$ at the last time to obtain the forgetting information, as shown in equation 14 .

$$
f_{t}=\sigma\left(W_{f}\left[h_{t-1}, x_{t}\right]+b_{f}\right)
$$

where, $\sigma$ is the global learning rate, $x_{t}$ is the current input

data, $h_{t-1}$ is the hidden information of the last time.

The input gate shall retain information according to the input layer information and hidden layer information, as shown in equation 15 .

$$
i_{t}=\sigma\left(W_{i}\left[h_{t-1}, x_{t}\right]+b_{i}\right)
$$

Long memory parameter update. The operation of the input gate should retain the information and update the long memory parameter $C$. In the previous step, the calculation of forgetting gate uses $C_{t-1}$, which only includes the previous information and does not reflect the input data at the current time. Update the long memory parameter $\mathrm{C}$, as shown in equation 16.

$$
C_{t}=\tanh \left(W_{C}\left[h_{t-1}, x_{t}\right]+b_{C}\right)
$$

$C_{t}$ values need to be updated not only with retained information but also with forgotten information. $C_{t-1}$ represents the forgotten information in the previous stage and $C_{t}$ is calculated as shown in equation 17.

$$
C_{t}=f_{t} C_{t-1}+i_{t} C_{t}^{C}
$$

Generate a new output, and the output calculation is shown in equation 18 .

$$
o_{t}=\sigma\left(W_{o}\left[h_{t-1}, x_{t}\right]+b_{o}\right)
$$

Use the output to update the short memory parameters, as shown in equation 19 .

$$
h_{t}=o_{t} \tanh \left(C_{t}\right)
$$

Next, based on the four-wheel steering system's hardware in the loop test platform, as shown in Figure 6, data acquisition is carried out to train neural network parameters. The controller of the bogie system communicates based on the CAN network. The controller forms data interaction with the upper computer software through NI data acquisition card and LabVIEW software. The upper computer constructs the vehicle dynamics model and traffic environment model through CarSim / Matlab software, forms the closed-loop hardware in the loop steering test, and obtains the test data under different road adhesion coefficients and different steering inputs.

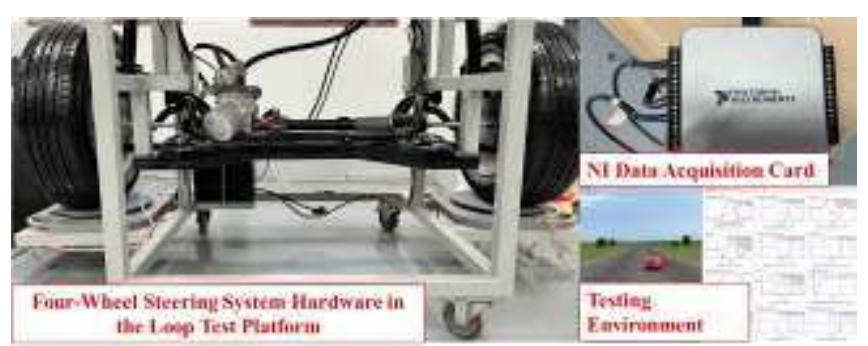

Fig. 6 Hardware in the loop test platform

The data acquisition test is carried out under double line change conditions under different initial conditions. The specific working condition settings in the test process are shown in Table 1. After each test, save the test data. In particular, the test data of side slip instability are also saved. Due to instability, the invalid data after the simulation stops are eliminated and used for LSTM estimator training.

Table 1 Test steps for data acquisition of cornering stiffness estimation 


\begin{tabular}{ccccc}
\hline $\begin{array}{c}\text { Test } \\
\text { steps }\end{array}$ & $\begin{array}{c}\text { Tire-road } \\
\text { friction } \\
\text { coefficient }\end{array}$ & Simulated speed & $\begin{array}{c}\text { Sampling } \\
\text { period (s) }\end{array}$ & $\begin{array}{c}\text { Test } \\
\text { termination } \\
\text { conditions }\end{array}$ \\
\hline 1 & 1.0 & Before the simulation, & 0.001 & $\begin{array}{c}1 . \text { The } \\
\text { driving }\end{array}$ \\
2 & 0.9 & set the simulation speed & 0.001 & distance of \\
3 & 0.8 & as 60km / h, 70km / h, & 0.001 & the vehicle \\
4 & 0.7 & 80km / h, 90km / h, & 0.001 & exceeds \\
5 & 0.6 & 100km / h, 110km / h & 0.001 & $200 \mathrm{~m} ;$ \\
6 & 0.5 & and $120 \mathrm{~km} / \mathrm{h}$ in & 0.001 & 2. The \\
7 & 0.4 & sequence. After all & 0.001 & simulation \\
8 & 0.3 & simulation speeds are & 0.001 & time exceeds \\
9 & 0.2 & completed, record them & 0.001 & 20 seconds; \\
\hline
\end{tabular}

The sample data obtained from the above tests are brought into the neural network for training, and the training results are compared with the basic LSTM algorithm and the LSTM based on the existing AdaGrad algorithm. The index results are shown in Table 2. It can be seen that the adaptive LSTM neural network shows a good estimation effect in the training set test.

Table 2 Test steps for data acquisition of cornering stiffness estimation

\begin{tabular}{ccccc}
\hline Algorithms & RMSE1 & RMSE2 & R Squared1 & R Squared2 \\
\hline Basic & $3.63 \mathrm{e}+03$ & $3.18 \mathrm{e}+03$ & 0.73 & 0.71 \\
AdaGrad & $1.57 \mathrm{e}+03$ & $1.36 \mathrm{e}+03$ & 0.82 & 0.88 \\
This Work & 487.61 & 242.37 & 0.95 & 0.92 \\
\hline
\end{tabular}

Then, the CL in the network model is analyzed. Because the model predictive control itself has a high demand for computational force, if the output result of the EL in the previous section is assigned to the controller in real-time, it will put forward higher requirements for the real-time calculation. This section proposes the regional classification network of tire cornering characteristics based on the EL to balance the two above. The overall structure of the algorithm after introducing the regional classification network is shown in Figure 7. Its feature is that the whole connection classification layer is connected in series behind the EL, As shown in Figure $7 \mathrm{~b}$. The regional classification method of tire cornering characteristics is constructed using the cornering stiffness value output in real-time by the estimator. The classification results shown in Fig. 7C are obtained: linear domain, nonlinear domain, and saturation domain, respectively.

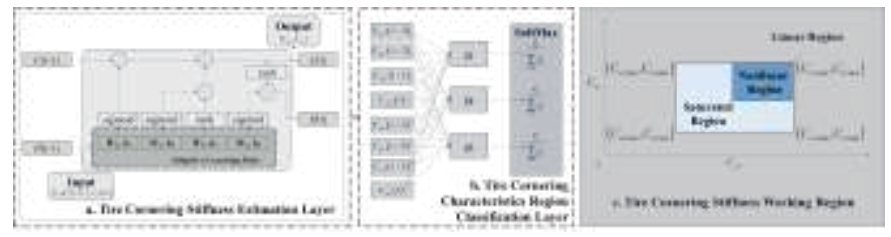

Fig. 7 Regional classification network model of tire cornering characteristics

The model adopts phased training. Firstly, the estimation layer is trained, and the regional classification layer is ignored. Then, when training the area classification part, lock the network parameters of the estimation part. The training results of the estimation part obtained separately are directly used for the training of area classification. According to the lateral stiffness value output by the current estimator, the original four-wheel steering system model is decomposed into three sub-models according to the linear region, nonlinear region, and saturation region. The working region of tire equivalent cornering stiffness comprises four vertices, $C_{1}\left(C_{\alpha f \max }, C_{\alpha r \max }\right), C_{2}\left(C_{\alpha f \text { max }}, C_{\alpha r \text { min }}\right), C_{3}\left(C_{\alpha f \text { min }}, C_{\alpha r \max }\right)$, and $C_{4}\left(C_{\alpha f \min }, C_{\alpha r \min }\right)$, as shown in Figure 7c. Where $C_{f \max }$ and $C_{r \max }$ are the maximum equivalent cornering stiffness of front wheel and rear wheel respectively; $C_{f \text { min }}$ and $C_{r \text { min }}$ are the minimum equivalent cornering stiffness of front wheel and rear wheel respectively.

4.2 Multi-model predictive stability control strategy for four-wheel steering system

To realize the stability control of the four-wheel steering system considering tire cornering characteristics, this section first studies the law between sideslip angle and stability region based on the phase plane method. It creatively introduces tire angle as the four-wheel steering system's phase plane stability region boundary under extreme working conditions to control the model predictive controller. On this basis, a multi-model predictive control (MMPC) strategy for a four-wheel steering system is proposed. According to the output from the CL in the network model, the sub-model parameters in the predictive controller are linear domain sub-model, nonlinear domain sub-model, and saturation domain sub-model, respectively. The stability control of the four-wheel steering system is effectively realized while ensuring calculation efficiency.

Yaw rate signal and sideslip angle signal are generally used to evaluate the stability of vehicles during steering [35, 36]. Among them, the yaw rate is determined by the yaw moment of the force on the tire to the centre of mass, and the resultant force determines the sideslip angle on the tire. Both of which are related to the force on the tire. It can be seen from equation 7 that there is a nonlinear coupling relationship between them. When the sideslip angle is slight, the yaw angle of the vehicle $\varphi$ is mainly determined by the vehicle yaw rate \&. Taking the front wheel steering vehicle as an example, the actual yaw rate that the vehicle system can achieve can be expressed as: 


$$
\underset{\text { real }}{\&}=\min \left\{\frac{v_{x}}{\left(1+K v_{x}^{2}\right) l} \delta_{f}, \frac{\mu g}{v_{x}}\right\}
$$

where, the former is the yaw rate that the front wheel angle can provide, and the latter is the yaw rate that can be achieved under the restriction of road adhesion coefficient. Under the two's constraints, the vehicle system's actual yaw rate can be obtained. Figure 8 below the solid line is the actual range of yaw rate that the vehicle system can achieve, and below the dotted line is the range of yaw rate that can be achieved under the restriction of road adhesion coefficient. The vehicle's actual yaw rate shall not exceed the yaw rate limit limited by the road adhesion conditions to maintain the vehicle's steering stability.

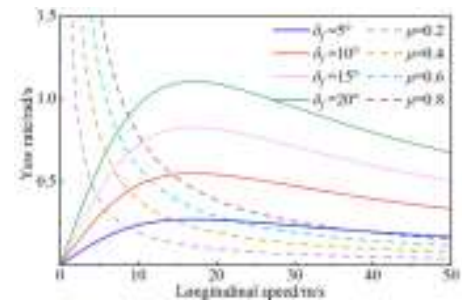

Fig. 8 Operating range of actual yaw rate

However, when the sideslip angle is large, its influence on the system's stability can not be ignored. As the included angle between the vehicle speed direction and the $\mathrm{x}$-axis direction of the body coordinate system, the sideslip angle will continue to increase with the increase of the lateral force. An excessive sideslip angle indicates that the lateral force tends to be saturated at this time. The curve is in the nonlinear region shown in Figure 3, and the system is close to the instability state. Many researchers have studied the relationship between yaw rate and system stability [37, 38]. However, compared with the yaw rate, the influence of sideslip angle on system stability is more complex. In this section, the effects of tire angle, longitudinal vehicle speed, and road adhesion coefficient on the parameters in the stability criterion are comprehensively considered to obtain the stability region represented by the sideslip angle, which provides a reference for the later design of controller constraints.

The critical phase trajectories of sideslip angle-yaw rate phase plane and sideslip angle-angular velocity phase plane under different working conditions are analyzed. According to the primary expression of the phase plane method for second-order nonlinear systems [39], The expression of the sideslip angle-yaw rate phase plane method is as follows.

$$
\left\{\begin{array}{l}
\beta=\frac{F_{Y f}+F_{Y r}}{m v_{x}}-\phi \\
I_{z}
\end{array}\right.
$$

Draw the phase plan of sideslip angle-yaw rate under different front wheel angles. Since the rear wheel angle is smaller than the front wheel angle, this section ignores the influence of the rear wheel angle on the phase plane stability region and focuses on the influence law of the front wheel angle on the system stability region. The longitudinal speed is set as $72 \mathrm{~km} / \mathrm{h}$, the road adhesion coefficient is 1 , and the front wheel angles are $0^{\circ}, 5^{\circ}, 10^{\circ}$, and $15^{\circ}$, respectively. The phased plan of sideslip angle-yaw rate and the boundary of stability region under different wheel angles are obtained, as shown in Figure 9. As shown in Figure 9, the stability region loses symmetry due to the increase of wheel angle, and the equilibrium point is no longer the zero point of the phase plane. It is not easy to express the stability coefficient of the wheel after the angle of the road is different from that of the road after the adjustment.

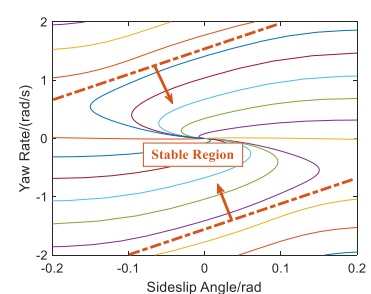

(a) $\delta_{f}=0^{\circ}$

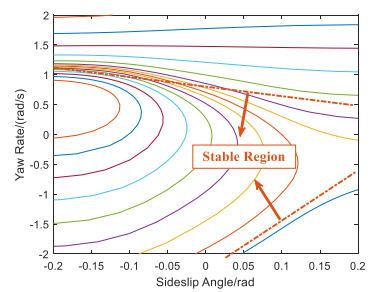

(c) $\delta_{f}=10^{\circ}$

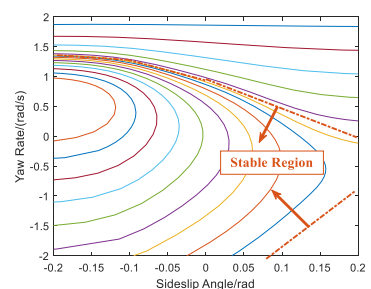

(b) $\delta_{f}=5^{\circ}$

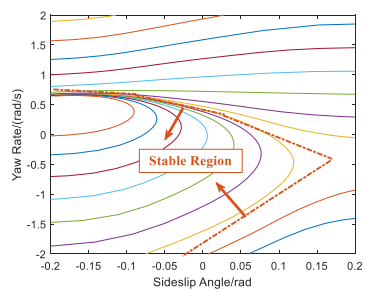

(d) $\delta_{f}=15^{\circ}$
Fig. 9 Phase plan and stability region boundary of sideslip angle-yaw rate at different wheel angles

Based on the phase plane diagram of sideslip angle yaw rate, the critical phase trajectory of sideslip angle-sideslip angular velocity phase plane under different front wheel angles is drawn by selecting the initial conditions near the stability region. Figure 10 shows the phase plan of sideslip angle - sideslip angular velocity under different wheel angles and the stability region boundary. With the increase of the front wheel angle, the slope of the right boundary does not change significantly. The slope of the left boundary is slightly larger than when the wheel angle is $0^{\circ}$, and the intersection of the lower boundary of the stability region and the longitudinal axis of the phase plane is significantly higher than that when the wheel angle is $0^{\circ}$. As shown from Figure 10, compared with the phase plan of sideslip angle yaw rate, although the stability region boundary of the phase plan of sideslip angle sideslip angular velocity also loses symmetry, it can still be divided by two oblique lines. It indicates that after introducing 
the influencing factors of wheel angle, sideslip angle - sideslip angular velocity phase plan can maintain a sound effect of characterizing the system's stability.

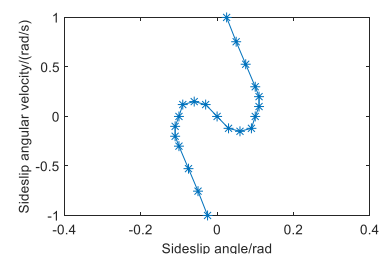

(a) $\delta_{f}=0^{\circ}$

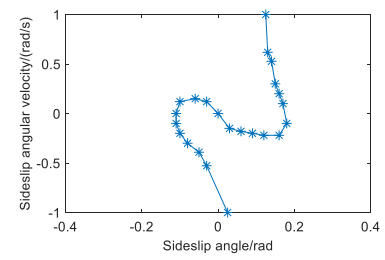

(c) $\delta_{f}=10^{\circ}$

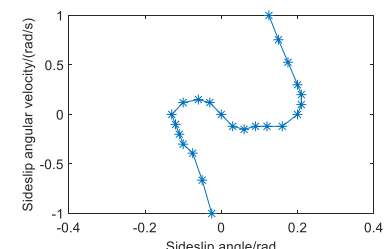

(b) $\delta_{f}=5^{\circ}$

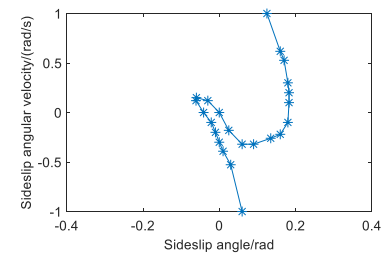

(d) $\delta_{f}=15^{\circ}$
Fig. 10 Sideslip angle sideslip angular velocity phase plane critical phase trajectory at different wheel angles

Similarly, the phase plan under different road adhesion coefficients and different longitudinal speeds can be obtained. By analyzing the above results, it can be concluded that: 1) the slope of the stability region boundary of the phase plane of the sideslip angle is related to the road adhesion coefficient, 2) the intersection of the stability region boundary and the coordinate axis is related to the longitudinal speed, 3) the intersection of the lower boundary of the stability region and the coordinate axis is also related to the front wheel angle. The influence of the front wheel angle on the slope of the lower boundary of the stability region is ignored. Three factors are integrated to describe the linear sideslip angle - sideslip angular velocity phase plane stability region. The determination method of phase plane stability region is obtained by fitting, considering road adhesion coefficient, longitudinal speed, and front-wheel angle, as shown in equation 22 .

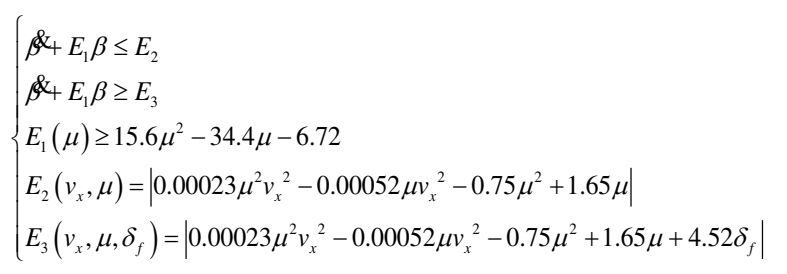

Compared with the existing research, the method considering the influence of the front-wheel angle proposed in this paper can more objectively reflect the dynamic parameter range in the steady-state under the actual working conditions and provide constraints for designing a four-wheel steering stability controller under extreme working conditions.

Then, a multi-model predictive control (MMPC) strategy for a four-wheel steering system is proposed, as shown in Figure 11. According to the working area of tire cornering stiffness output by the classification layer in the network model, the controller switches the parameters of its sub-model, which are linear domain sub-model, nonlinear domain sub-model, and saturation domain sub-model, respectively. The stability control of the four-wheel steering system under the condition of low adhesion coefficient is effectively realized while ensuring calculation efficiency. The equivalent lateral stiffness of the front and rear wheels in the linear domain sub-model predictive controller is the maximum equivalent lateral stiffness of the front and rear wheels, and the value is $C_{a f}=66900 \mathrm{~N} / \mathrm{rad}, C_{\alpha r}=62700 \mathrm{~N} / \mathrm{rad}$. The equivalent lateral stiffness of the front and rear wheels in the nonlinear working domain sub-model predictive controller is $C_{\alpha f}=44154 \mathrm{~N} / \mathrm{rad}, C_{\alpha r}=41382 \mathrm{~N} / \mathrm{rad}$. The equivalent lateral stiffness of the front and rear wheels in the saturated working domain sub-model predictive controller is $C_{\alpha f}=30364 \mathrm{~N} / \mathrm{rad}$, $C_{\alpha r}=28466 \mathrm{~N} / \mathrm{rad}$.

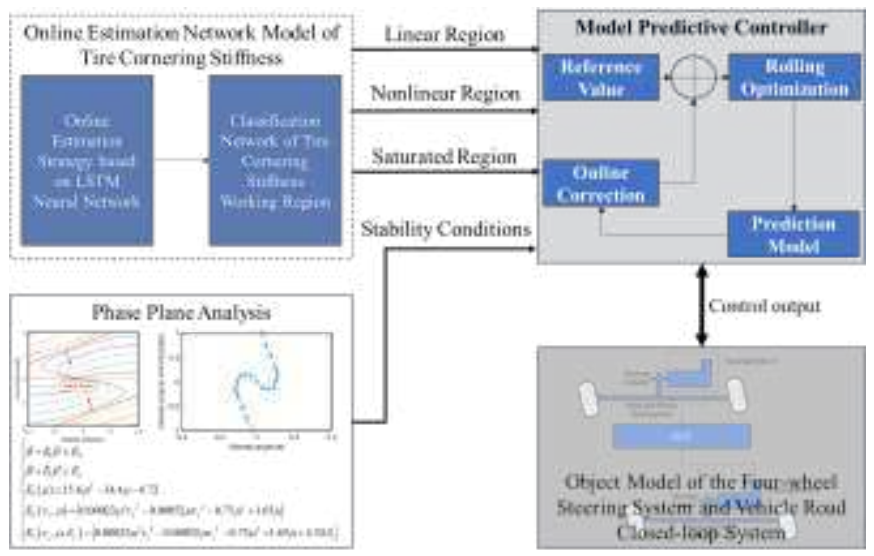

Fig. 11 Flow chart of model predictive control strategy for four-wheel steering system

Next, the prediction model and stability constraints under limit conditions are established. Consider the nonlinear dynamic model as shown in equation 23 .

$$
\psi \&=f(\psi, u)
$$

The selected state quantity is shown in equation 24 .

$$
\psi \&=\left[\begin{array}{llllll}
Y & X & \& & \& & \varphi & \phi
\end{array}\right]^{T}
$$

The selected control quantity is shown in equation 25 .

$$
u=\left[\begin{array}{ll}
\delta_{f} & \delta_{r}
\end{array}\right]^{T}
$$

The controller realizes trajectory tracking by controlling the front-wheel and rear-wheel angles. This section mainly analyses the lateral dynamics, so the longitudinal speed of the vehicle remains unchanged. The current working point is the equilibrium point for linearisation expansion, and the Jacobian matrix approximately describes the dynamic model established in Section 3. The linearized dynamic model can be obtained by expansion. 


$$
\psi \&=A(t) \psi(t)+B(t) u(t)
$$

where,

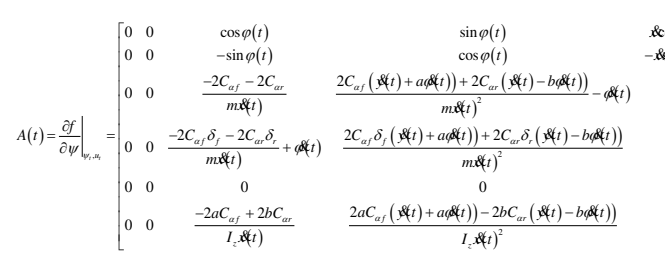

$$
\begin{gathered}
x \cos \varphi(t)-x \sin \varphi(t) \\
-x \sin \varphi(t)-\cos \varphi(t) \\
0 \\
0 \\
0
\end{gathered}
$$$$
B(t)=\left.\frac{\partial f}{\partial u}\right|_{\psi_{,}, u_{t}}=\left[\begin{array}{cc}
0 & 0 \\
0 & 0 \\
\frac{2 C_{\alpha f}}{m} & \frac{2 C_{\alpha r}}{m} \\
\frac{2 C_{\alpha f}\left(2 \delta_{f}-\frac{\&(t)+a(\&(t))}{m \&(t)}\right)}{0} & \frac{2 C_{\alpha r}\left(2 \delta_{r}-\frac{\&(t)-b r \&(t)}{m \& t)}\right)}{m} \\
\frac{2 a C_{\alpha f}}{I_{z}} & \frac{-2 b C_{\alpha r}}{I_{z}}
\end{array}\right]
$$

Equation 25 is discretized by the zero-order holding method. The discrete model can be expressed as shown in equation 27.

$$
\begin{aligned}
& x(k+1)=A_{2} x(k)+B_{2} u(k) \\
& y(k)=C_{2} x(k)
\end{aligned}
$$

where,

$$
\begin{aligned}
& A_{2}=\Phi(T)=e^{A_{1} T}=\sum_{i=0}^{\infty} \frac{A_{1}^{i} T^{i}}{i !} \\
& B_{2}=\int_{0}^{T} \Phi\left(\tau^{\prime}\right) B_{1} d \tau^{\prime}=B_{1} \cdot \int_{0}^{T} e^{A_{1} \tau^{\prime}} d \tau^{\prime}=B_{1} \cdot \sum_{i=0}^{\infty} \frac{A_{1}^{i} T^{i+1}}{(i+1) !} \\
& C_{2}=C_{1}
\end{aligned}
$$

Considering that in practical application, the front wheel angle, rear-wheel angle, front-wheel angle increment, and rear-wheel angle increment cannot exceed the physical limit, the control increment is introduced into the prediction model. The original dynamic model is expanded, and the control increment is introduced. The expanded dynamic model can be expressed as:

$$
\begin{aligned}
& \xi(k+1 \mid k)=\not \mathcal{\xi}(k \mid k)+B \Delta u(k \mid k) \\
& \eta(k+1 \mid k)=\mathscr{E} \xi(k+1 \mid k)
\end{aligned}
$$

where, $\xi(k+1 \mid k)=\left[\begin{array}{c}\psi(k+1 \mid k) \\ u(k \mid k)\end{array}\right], \quad \eta(k+1 \mid k)=\psi(k+1 \mid k)$,

$$
\mathscr{A}=\left[\begin{array}{cc}
A_{2} & B_{2} \\
0 & I
\end{array}\right], \quad B=\left[\begin{array}{c}
B_{2} \\
I
\end{array}\right], \quad \&=\left[\begin{array}{llllll}
1 & 0 & 0 & 0 & 0 & 0 \\
0 & 1 & 0 & 0 & 0 & 0 \\
0 & 0 & 1 & 0 & 0 & 0 \\
0 & 0 & 0 & 1 & 0 & 0
\end{array}\right]
$$

After further iteration, the control sequence in the predictive horizon is obtained, which can be expressed as shown in equation 29.

$$
\Phi(k)=Z \xi(k)+\Theta \Delta U(k)
$$

where,

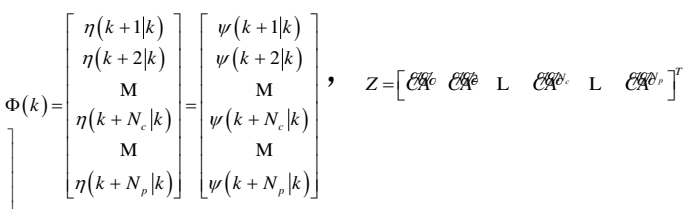

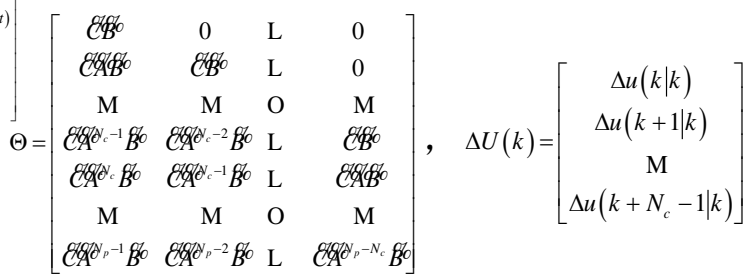

After the prediction model is obtained, the objective function is constructed. The objective function should ensure that the vehicle can track the reference path quickly and stably. Therefore, the objective function needs to include the state quantity and control increment parts to optimize the path tracking performance. The constructed objective function can be expressed as shown in equation 30 .

$$
\begin{aligned}
& J=\sum_{i=1}^{p}\|(\Phi(k-1+i \mid k)-R(k-1+i \mid k))\|_{P}^{2} \\
& +\sum_{i=1}^{c}\|\Delta U(k-1+i \mid k)\|_{Q}^{2}+\rho \varepsilon^{2}
\end{aligned}
$$

where, the first item reflects the tracking ability of the system to the reference trajectory, the second item reflects the requirements for the smooth change of the control quantity, and the third item is the relaxation factor to avoid the situation that the controller cannot obtain the optimal solution.

The stability constraints under extreme conditions are designed below. Constrain the front wheel angle, rear-wheel angle, front-wheel angle increment, and rear-wheel angle increment.

$$
\begin{aligned}
& {\left[\begin{array}{c}
-25^{\circ} \\
-10^{\circ}
\end{array}\right] \leq\left[\begin{array}{l}
\delta_{f} \\
\delta_{r}
\end{array}\right] \leq\left[\begin{array}{c}
25^{\circ} \\
10^{\circ}
\end{array}\right]} \\
& {\left[\begin{array}{c}
-5^{\circ} \\
-5^{\circ}
\end{array}\right] \leq\left[\begin{array}{c}
\Delta \delta_{f} \\
\Delta \delta_{r}
\end{array}\right] \leq\left[\begin{array}{l}
5^{\circ} \\
5^{\circ}
\end{array}\right]}
\end{aligned}
$$

Through the analysis of the phase plane of the sideslip angle-sideslip angle velocity, it can be obtained that the sideslip angle significantly influences the vehicle's stability. With the decrease of the road adhesion coefficient, the range of the phase plane stability domain gradually narrows. Therefore, it is necessary to introduce the upper and lower bounds of the phase plane stability domain of the centroid sideslip angle and the centroid sideslip angle velocity obtained in Section 3 into the constraints. The sideslip angle is used as a stability constraint, and the state quantity based on the prediction model can be expressed in equation 32 .

$$
\beta=\frac{\not \&}{\not \&}
$$

The vehicle's dynamic performance is restricted by the 
driving force and the adhesion conditions between the tire and the ground. Therefore, it is necessary to add constraints on the adhesion conditions of the vehicle. Longitudinal and lateral accelerations are limited by ground adhesion.

$$
\sqrt{a_{x}^{2}+a_{y}^{2}} \leq \mu g
$$

where, $a_{x}$ is the longitudinal acceleration and $a_{y}$ is the lateral acceleration. The stability constraint for the lateral acceleration can be expressed in equation 34 .

$$
-\mu g \leq a_{y} \leq \mu g
$$

The state quantity of lateral acceleration based on the prediction model can be expressed in equation 35 .

$$
\begin{aligned}
& a_{y}=\frac{v_{x}^{2}}{R}=\frac{-2 C_{\alpha f}-2 C_{\alpha r}}{m v_{x}} \&+\left(\frac{-2 a C_{\alpha f}+2 b C_{\alpha r}}{m v_{x}}-v_{x}\right) \& \& \\
& +\frac{2 C_{\alpha f}}{m} \delta_{f}+\frac{2 C_{\alpha r}}{m} \delta_{r}+v_{x} \& \&
\end{aligned}
$$

Considering that the constraints are too narrow, there will be problems such as solution failure, and the optimal solution cannot be obtained, so the tire sideslip constraint is set as a soft constraint, and the constraint factor is added in the formula. The solver dynamically adjusts the constraint according to the solution of each control cycle. Based on the prediction model, the state quantity of tire sideslip angle can be expressed in equation 36 .

$$
\alpha_{r}=\frac{\& b \phi \&}{v_{x}}-\delta_{r}
$$

Finally, the constraints of the model predictive controller considering the stability of extreme working conditions can be expressed in equation 37 .

$$
\begin{gathered}
u_{\text {min }} \leq u \leq u_{\max } \\
\Delta u_{\text {min }} \leq \Delta u \leq \Delta u_{\text {max }} \\
a_{y \text { min }} \leq a_{y} \leq a_{y \text { max }} \\
\alpha_{r \text { min }}-\varepsilon \leq \alpha_{r} \leq \alpha_{r \text { max }}+\varepsilon \\
\beta_{\text {min }} \leq \beta \leq \beta_{\text {max }}
\end{gathered}
$$

The final objective function is transformed into the general form of quadratic programming [40].

\section{Results and discussion}

This section designs the hardware in the loop test platform to verify the effectiveness of the multi-model predictive stability control strategy proposed above. In addition to the embedded steering controller, the test hardware in the loop simulation system is also equipped with an upper computer simulation system composed of NI PXI and two personnel computers, as shown in Figure 12. The embedded steering controller uses NXP 5744 as the main control chip. NI PXI and computers are used to construct vehicle simulation environments and the real-time solution of the controller model. The embedded steering controller interacts with the upper computer simulation system based on the CAN bus and collects data through the can card built-in Ni PXI. In addition, during the test, the data is collected through PCAN to verify the test data, which is convenient for the data analysis after the system reports an error.
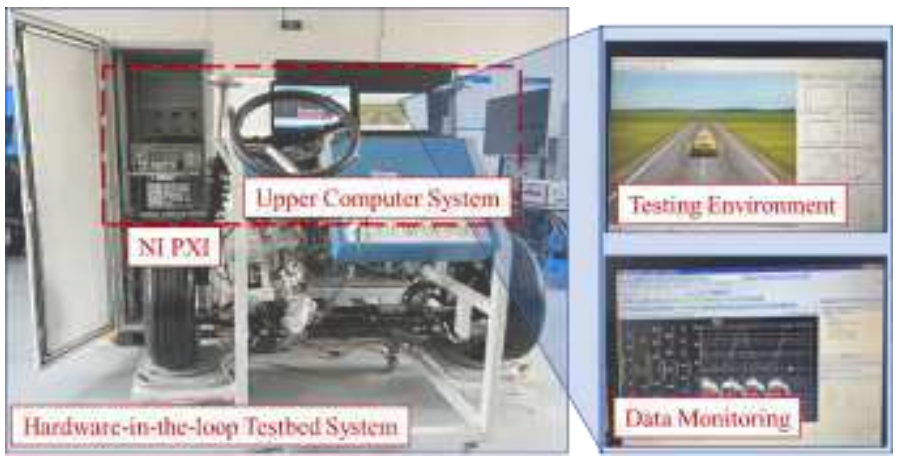

Fig. 12 Four-wheel steering vehicle hardware in the loop test platform

The main parameters of the four-wheel steering system hardware in the loop test platform are shown in Table 3.

Table 3 Main parameters of hardware in the loop test platform for four-wheel steering system

\begin{tabular}{cccc}
\hline Parameter & Symbol & Value & Unit \\
\hline Armature inductance of steering motor & $L$ & 126 & $\mathrm{mH}$ \\
Armature resistance of steering motor & $R$ & 16.8 & $\mathrm{~m} \Omega$ \\
Back EMF constant of the steering motor & $K_{\mathrm{b}}$ & 5.99 & $\mathrm{~V} / \mathrm{Krpm}$ \\
Mass of rack & $M_{r}$ & 8.5 & $\mathrm{~kg}$ \\
Damping coefficient of rack & $B_{r}$ & 35.4 & $\mathrm{~N} /(\mathrm{m} / \mathrm{s})$ \\
Pinion radius & $N$ & 20 & $\backslash$ \\
The reduction ratio of the steering motor & $r_{p}$ & 82 & $\mathrm{~mm}$ \\
Distance from the centroid to the front & $\mathrm{a}$ & 1.4 & $\mathrm{~m}$ \\
Distance from the centroid to rear axle & $\mathrm{b}$ & 1.6 & $\mathrm{~m}$ \\
Moment of inertia of the system about & & & \\
the z-axis & $I_{\mathrm{z}}$ & &
\end{tabular}

When driving at high speed with a low friction coefficient, steering behaviours like emergency avoidance often lead to vehicle instability. Therefore, reasonable extreme test conditions are constructed based on steering driving conditions such as double lane change (DLC). The lateral reference position and reference heading angle under double line shifting conditions can be expressed in equation 38 .

$$
\left\{\begin{array}{l}
Y_{r e f}(X)=\frac{d_{y 1}}{2}\left(1+\tanh \left(z_{1}\right)\right)-\frac{d_{y 2}}{2}\left(1+\tanh \left(z_{2}\right)\right) \\
\varphi_{r e f}(X)=\arctan \left(d_{y 1}\left(\frac{1}{\cosh \left(z_{1}\right)}\right)^{2}\left(\frac{1.2}{d_{x 1}}\right)-d_{y 2}\left(\frac{1}{\cosh \left(z_{2}\right)}\right)^{2}\left(\frac{1.2}{d_{x 2}}\right)\right)
\end{array}\right.
$$

where, $\quad z_{1}=\frac{2.4}{25}(X-27.19)-1.2 \quad ; \quad z_{2}=\frac{2.4}{21.95}(X-56.46)-1.2 \quad$;

$d_{x 1}=25 ; \quad d_{x 2}=21.95 ; \quad d_{y 1}=4.05 ; \quad d_{y 2}=5.7$ 。

The reference front and rear wheel angles of the 
four-wheel steering system during the implementation of the DLC condition are shown in Figure 13a, and the reference yaw angle under the DLC condition is shown in Figure $13 \mathrm{~b}$.

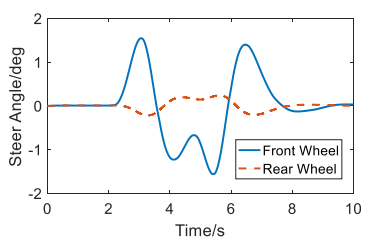

a. The reference front and rear wheel angles

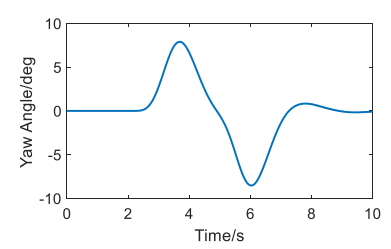

b. The reference yaw angle
Fig. 13 Reference wheel angle and reference yaw angle under DLC condition

The parameter values of the multi-model predictive controller during the test are shown in Table 4.

Table. 4 Controller parameters

\begin{tabular}{cc}
\hline Parameter & Value \\
\hline Sampling time / s & 0.02 \\
Prediction Horizon & 20 \\
Control Horizon & 5 \\
\hline
\end{tabular}

\subsection{Discussion on steering tracking performance}

It can be seen from the analysis in Section 3 that a lower road adhesion coefficient will reduce not only the maximum value of tire lateral force but also reduce the sideslip angle corresponding to the limit of tire lateral force. The nonlinear region of tire sideslip characteristics will increase with the decrease of road adhesion coefficient, resulting in the risk of sideslip of the four-wheel steering system. Therefore, the tracking effect of the DLC process when the road adhesion coefficient is 0.2 and 0.5 is tested and compared, and the simulation speed is $80 \mathrm{~km} / \mathrm{h}$.

The tracking control effects of the two algorithms when the adhesion coefficient is 0.5 are compared, as shown in Figure 14. Compared with the basic MPC algorithm, the maximum deviation of the front wheel angle and rear wheel angle based on the controller designed in this paper are reduced by $35.15 \%$ and $67.94 \%$. The comparison between the heading angle obtained based on the two controllers and the reference yaw angle is shown in Fig. 14C. The simulation results show that the maximum deviation between the controller designed in this paper and the reference yaw angle is $1.329 \mathrm{deg}$, reduced by $65.96 \%$ compared with the basic MPC algorithm. At this time, the reference value can still be tracked by both algorithms.

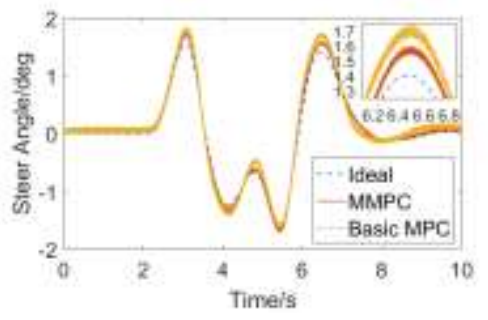

a. Front-wheel angle

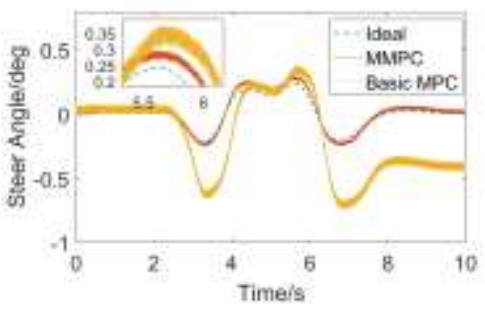

b. Rear-wheel angle

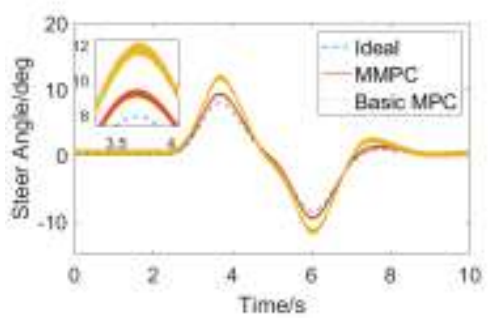

c. Yaw angle

Fig. 14 Comparison of tracking effect in DLC process $(\mu=0.5)$

The tracking control effect of the two algorithms is further compared when the adhesion coefficient is 0.2 , as shown in Figure 15. It can be obtained that the mean square error wheel angles of the front and rear deviations of the controller designed in this paper and the reference are 12.66 degrees and 0.12 degrees, respectively, and eventually converge gradually after oscillation and will not enter an unstable state. However, the front and rear wheel angles obtained based on the basic MPC algorithm gradually increase, and the tracking cannot be completed. The comparison between the yaw angle obtained based on the two algorithms and the reference yaw angle is shown in Figure 15c. Based on the algorithm proposed in this paper, the mean square error between the result and the reference yaw angle is $10.32 \mathrm{deg}$. In contrast, the algorithm based on this paper can still track the reference tire steering angle and reference yaw angle under the condition of low road adhesion coefficient. The results obtained with basic MPC have tracking lag and cannot complete the tracking.

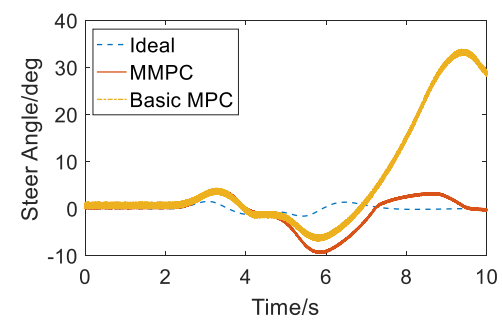

a. Front-wheel angle

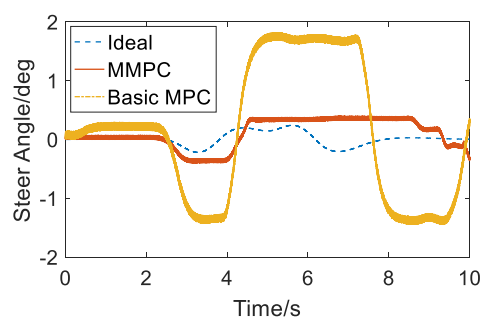

b. Rear-wheel angle 


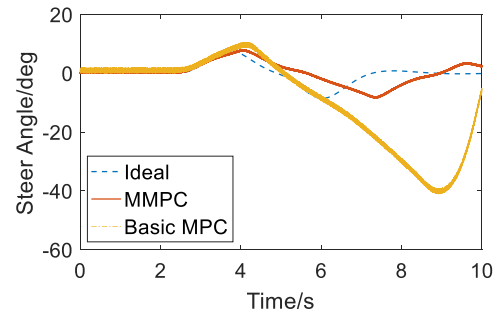

c. Yaw angle

Fig. 15 Comparison of tracking effect in DLC process $(\mu=0.2)$

The causes of the above results are further discussed, and the variation of tire cornering force cornering angle curve with time under two working conditions is obtained, as shown in Figure 16. Compared with the results obtained by the basic MPC algorithm, the MMPC algorithm proposed in this paper can obtain the greater lateral force of front and rear wheels. When the sideslip instability of the system is about to occur due to the tire cornering characteristics, the system's stability is ensured by switching the sub-model.

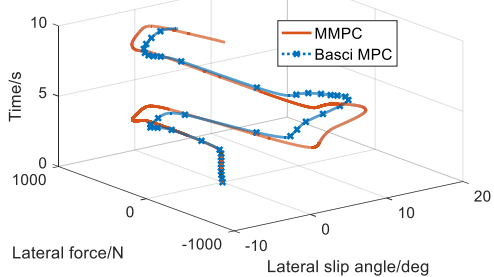

a. Front-wheel angle

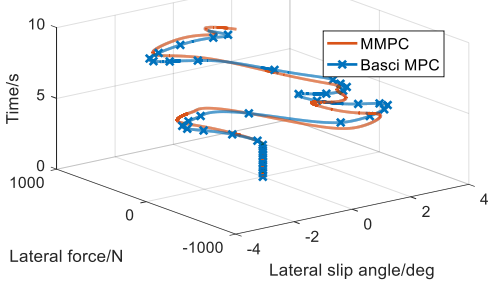

b. Rear-wheel angle

Fig. 16 Comparison of front and rear wheel lateral force-sideslip angle curves in DLC process

\subsection{Discussion on system stability}

Based on the test designed in the previous section, the system's stability in the test process is further compared and analyzed. Figure 17 shows the comparison of the side deflection angle of the centroid of the two controllers when the road adhesion coefficient is 0.5 and 0.2 . The sorting results are shown in Table 5. The above results are discussed. When the adhesion coefficient is 0.5 , the sideslip angle of the four-wheel steering vehicle system based on the controller designed in this paper is smaller, and the MSE index is $57.69 \%$ lower than the basic MPC. When the adhesion coefficient is 0.2 , it can be found that there is an apparent boundary on the sideslip angle curve, which shows the effectiveness of the sideslip angle constraint in the model predictive controller and ensures the stability of the system.

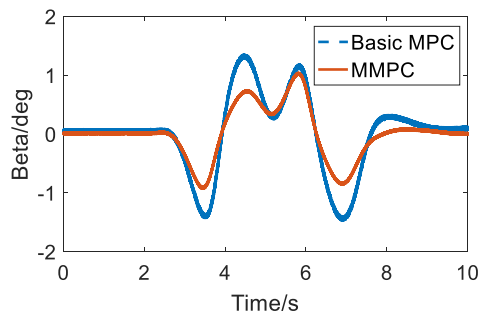

a. $\mu=0.5$

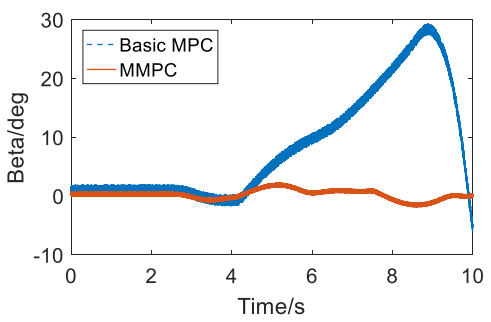

b. $\mu=0.2$

Fig. 17 Comparison of sideslip angle in the process of DLC

Table. 5 Analysis of the results of sideslip angle in the process of DLC

\begin{tabular}{ccc}
\hline Algorithm & $\operatorname{MSE}(\mu=0.5)$ & $\operatorname{MSE}(\mu=0.2)$ \\
\hline Basic MPC & 0.4167 & 140.1772 \\
MMPC & 0.1763 & 0.6161 \\
\hline
\end{tabular}

The time-dependent phase diagrams of important dynamic parameters such as yaw rate, sideslip angle, and lateral acceleration obtained by the two algorithms during the test are further discussed. Compared with the basic MPC algorithm, the phase diagram of dynamic parameters obtained based on the MMPC algorithm converges faster with time, and when the MMPC algorithm is used in the test process, the dynamic parameters are smaller in both change rate and peak value, that is, the system is easier to enter the stable state. Thus, the effectiveness of the proposed algorithm instability control is further illustrated.

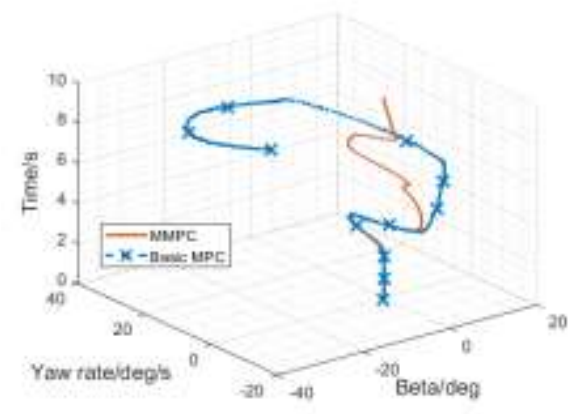

a. Beta- Yaw rate

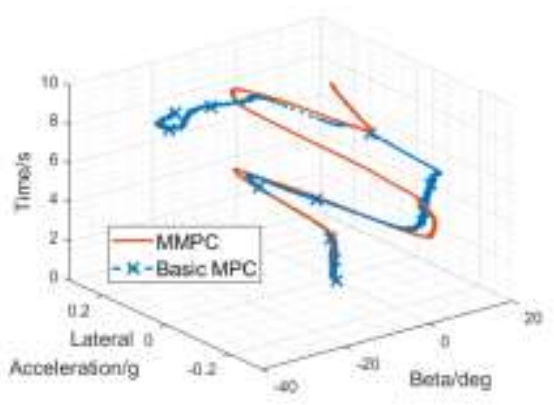

b. Beta- Lateral acceleration 


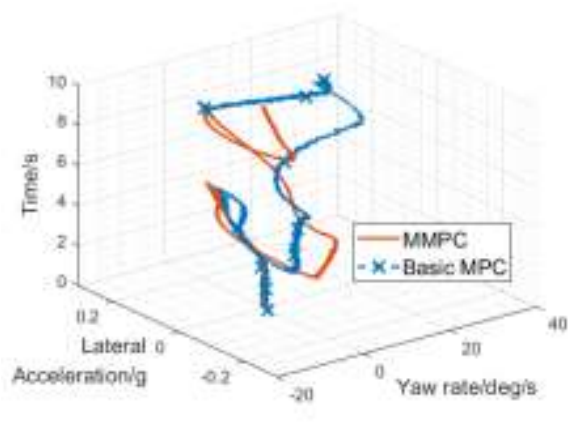

c. Yaw rate- Lateral acceleration

Fig. 18 Phase diagram comparison of kinetic parameters

\section{Conclusions}

This paper proposes a cascade deep learning framework combining multi-model predictive control (MMPC) and LSTM tire cornering stiffness estimation (TCSE) neural network and designs a stability control strategy of vehicle four-wheel steering system considering tire nonlinear cornering characteristics. In the rolling optimization process of MMPC, the controller switches the sub-model parameters in MMPC according to the current working domain of the tire output by the TCSE network. Compared with the existing research, this strategy balances the contradiction between computational efficiency and model accuracy.

In addition, this paper established a hardware-in-the-loop simulation platform to collect data sets used for network model parameter training and verify the algorithm's effectiveness. The test results show that, in terms of steering tracking performance, compared with the Basic MPC algorithm, when the adhesion coefficient is 0.5 , the maximum deviations between the front and rear wheel angles and heading angles from the ideal values are reduced by $35.15 \%$, $67.94 \%$, and $67.94 \%$, respectively. $65.96 \%$. When the adhesion coefficient is 0.2 , the method proposed in this paper can still ensure the system tracks the ideal value, while the results obtained by the basic MPC algorithm can not be tracked. The MSE index of sideslip angle obtained by the algorithm proposed in this paper is $57.69 \%$ lower than basic MPC in stability control. When the adhesion coefficient is 0.2 , the sideslip angle curve obtained by the method proposed in this paper has an obvious boundary, which shows the effectiveness of the sideslip angle constraint in the model predictive controller and ensures the system's stability.

Next, on the one hand, further analyze the influence of other influencing factors other than the front wheel angle, longitudinal speed, and road adhesion coefficient on the system stability control, and explore its influence law. On the other hand, we explore higher precision estimation algorithms of tire cornering stiffness and road adhesion coefficient to improve control accuracy by studying different estimation algorithms.

\section{Acknowledgements}

This research presented within this article was supported by the National Nature Science Foundation of China (Grant No. 52072175 and Grant No. 51875279), the Postgraduate Research and Practice Innovation Program of Jiangsu Province KYCX21_0186.

\section{Data Availability Statement}

Data will be made available on reasonable request.

\section{Declaration}

Conflict of interest The authors declare that they have no conflict of interest.

\section{References}

1. Di Cairano, S., Tseng, H.E., Bernardini, D., Bemporad, A.: Vehicle Yaw Stability Control by Coordinated Active Front Steering and Differential Braking in the Tire Sideslip Angles Domain. IEEE Trans. Contr. Syst. Technol. 21, 1236-1248 (2013). https://doi.org/10.1109/TCST.2012.2198886

2. Bevly, D.M., Ryu, J., Gerdes, J.C.: Integrating INS Sensors With GPS Measurements for Continuous Estimation of Vehicle Sideslip, Roll, and Tire Cornering Stiffness. IEEE Trans. Intell. Transport. Syst. 7, 483-493 https://doi.org/10.1109/TITS.2006.883110

(2006).

3. Kanghyun Nam, Fujimoto, H., Hori, Y.: Lateral Stability Control of In-Wheel-Motor-Driven Electric Vehicles Based on Sideslip Angle Estimation Using Lateral Tire Force Sensors. IEEE Trans. Veh. Technol. 61, 1972-1985 (2012). https://doi.org/10.1109/TVT.2012.2191627

4. Guo, J., Luo, Y., Li, K.: Dynamic coordinated control for over-actuated autonomous electric vehicles with nonholonomic constraints via nonsingular terminal sliding mode technique. Nonlinear Dyn. 85, 583-597 (2016). https://doi.org/10.1007/s11071-016-2708-2

5. Kang, C.M., Lee, S.-H., Chung, C.C.: Discrete-Time LPV Observer With Nonlinear Bounded Varying Parameter and Its Application to the Vehicle State Observer. IEEE Trans. Ind. Electron. 65, 8768-8777 (2018). https://doi.org/10.1109/TIE.2018.2813961

6. Ye, Y., He, L., Zhang, Q.: Steering Control Strategies for a Four-Wheel-Independent-Steering Bin Managing Robot. IFAC-PapersOnLine. 49, 39-44 (2016). https://doi.org/10.1016/j.ifacol.2016.10.008

7. Jiang, L., Wang, S., Meng, J., Zhang, X., Jin, J., Xie, Y.: Inverse Decoupling-based Direct Yaw Moment Control of a Four-wheel Independent Steering Mobile Robot. In: 2020 IEEE/ASME International Conference on Advanced Intelligent Mechatronics (AIM). pp. 892-897. 
IEEE, Boston, MA, USA (2020)

8. Zhou, C., Liu, X., Xu, F.: Intervention criterion and control strategy of active front steering system for emergency rescue vehicle. Mechanical Systems and Signal Processing. 148, $107160 \quad$ (2021). https://doi.org/10.1016/j.ymssp.2020.107160

9. Cao, M., Hu, C., Wang, J., Wang, R., Chen, N.: Adaptive complementary filter-based post-impact control for independently-actuated and differentially-steered autonomous vehicles. Mechanical Systems and Signal Processing. 144, 106852 (2020). https://doi.org/10.1016/j.ymssp.2020.106852

10. Chen, T., Chen, L., Xu, X., Cai, Y., Jiang, H., Sun, X.: Passive fault-tolerant path following control of autonomous distributed drive electric vehicle considering steering system fault. Mechanical Systems and Signal Processing. 123, 298-315 (2019). https://doi.org/10.1016/j.ymssp.2019.01.019

11. Taghavifar, H., Hu, C., Taghavifar, L., Qin, Y., Na, J., Wei, C.: Optimal robust control of vehicle lateral stability using damped least-square backpropagation training of neural networks. Neurocomputing. 384, 256-267

(2020). https://doi.org/10.1016/j.neucom.2019.12.045

12. Chatzikomis, C.I., Spentzas, K.N.: Comparison of a vehicle equipped with Electronic Stability Control (ESC) to a vehicle with Four Wheel Steering (4WS). Forsch Ingenieurwes. 78, 13-25 (2014). https://doi.org/10.1007/s10010-014-0172-z

13. Hang, P., Chen, X., Fang, S., Luo, F.: Robust control for four-wheel-independent-steering electric vehicle with steer-by-wire system. Int.J Automot. Technol. 18, 785-797

(2017).

https://doi.org/10.1007/s12239-017-0078-5

14. Farroni, F., Russo, M., Russo, R., Terzo, M., Timpone, F.: A combined use of phase plane and handling diagram method to study the influence of tyre and vehicle characteristics on stability. Vehicle System Dynamics. 51, 1265-1285 (2013). https://doi.org/10.1080/00423114.2013.797590

15. Zhang, J., Wang, H., Ma, M., Yu, M., Yazdani, A., Chen, L.: Active Front Steering-Based Electronic Stability Control for Steer-by-Wire Vehicles via Terminal Sliding Mode and Extreme Learning Machine. IEEE Trans. Veh. Technol. 69, 14713-14726 (2020). https://doi.org/10.1109/TVT.2020.3036400

16. Ding, S., Liu, L., Zheng, W.X.: Sliding Mode Direct Yaw-Moment Control Design for In-Wheel Electric Vehicles. IEEE Trans. Ind. Electron. 64, 6752-6762 (2017). https://doi.org/10.1109/TIE.2017.2682024

17. Zhang, H., Huang, X., Wang, J., Karimi, H.R.: Robust energy-to-peak sideslip angle estimation with applications to ground vehicles. Mechatronics. 30, 338-347 (2015). https://doi.org/10.1016/j.mechatronics.2014.08.003

18. Hu, C., Jing, H., Wang, R., Yan, F., Chadli, M.: Robust $\mathrm{H} \infty$ output-feedback control for path following of autonomous ground vehicles. Mechanical Systems and Signal Processing. 70-71, 414-427 (2016). https://doi.org/10.1016/j.ymssp.2015.09.017

19. Wang, T., Kang, Y., Li, P., Zhao, Y.-B., Yu, P.: Robust model predictive control for constrained networked nonlinear systems: An approximation-based approach. Neurocomputing. $\quad 418, \quad 56-65 \quad$ (2020). https://doi.org/10.1016/j.neucom.2020.07.037

20. Sarailoo, M., Rahmani, Z., Rezaie, B.: A novel model predictive control scheme based on bees algorithm in a class of nonlinear systems: Application to a three tank system. Neurocomputing. 152, 294-304 (2015). https://doi.org/10.1016/j.neucom.2014.10.066

21. Han, K., Choi, M., Choi, S.B.: Estimation of the Tire Cornering Stiffness as a Road Surface Classification Indicator Using Understeering Characteristics. IEEE Trans. Veh. Technol. 67, 6851-6860 (2018). https://doi.org/10.1109/TVT.2018.2820094

22. Xing, Y., Lv, C.: Dynamic State Estimation for the Advanced Brake System of Electric Vehicles by Using Deep Recurrent Neural Networks. IEEE Trans. Ind. Electron. 67, 9536-9547 (2020). https://doi.org/10.1109/TIE.2019.2952807

23. Zhang, Z., Zhang, X., Pan, H., Salman, W., Rasim, Y., Liu, X., Wang, C., Yang, Y., Li, X.: A Novel Steering System for a Space-Saving 4WS4WD Electric Vehicle: Design, Modeling, and Road Tests. IEEE Trans. Intell. Transport. Syst. 18, 114-127 (2017). https://doi.org/10.1109/TITS.2016.2561626

24. Hou, S., Xu, W., Liu, G.: Design of an Interacting Multiple Model-Cubature Kalman Filter Approach for Vehicle Sideslip Angle and Tire Forces Estimation. Mathematical Problems in Engineering. 2019, 1-13 (2019). https://doi.org/10.1155/2019/6087450

25. Hu, J.-S., Yin, D., Hori, Y.: Fault-tolerant traction control of electric vehicles. Control Engineering Practice. 19, 204-213 (2011). https://doi.org/10.1016/j.conengprac.2010.11.012

26. Demir, O., Keskin, I., Cetin, S.: Modeling and control of a nonlinear half-vehicle suspension system: a hybrid fuzzy logic approach. Nonlinear Dyn. 67, 2139-2151 (2012). https://doi.org/10.1007/s11071-011-0135-y

27. Zhao, L.-H., Liu, Z.-Y., Chen, H.: Design of a Nonlinear Observer for Vehicle Velocity Estimation and Experiments. IEEE Trans. Contr. Syst. Technol. 19, 664-672

https://doi.org/10.1109/TCST.2010.2043104

(2011).

28. Tahouni, A., Mirzaei, M., Najjari, B.: Novel Constrained Nonlinear Control of Vehicle Dynamics Using Integrated Active Torque Vectoring and Electronic Stability Control. IEEE Trans. Veh. Technol. 68, 9564-9572 (2019). https://doi.org/10.1109/TVT.2019.2933229

29. Vicente, B.A.H., James, S.S., Anderson, S.R.: Linear System Identification Versus Physical Modeling of Lateral-Longitudinal Vehicle Dynamics. IEEE Trans. Contr. Syst. Technol. 29, 1380-1387 (2021). https://doi.org/10.1109/TCST.2020.2994120

30. Davoodabadi, I., Ramezani, A.A., Mahmoodi-k, M., Ahmadizadeh, P.: Identification of tire forces using Dual Unscented Kalman Filter algorithm. Nonlinear Dyn. 78, 1907-1919 (2014). https://doi.org/10.1007/s11071-014-1566-Z

31. Ran, X., Shan, Z., Fang, Y., Lin, C.: An LSTM-Based Method with Attention Mechanism for Travel Time $\begin{array}{llll}\text { Prediction. } & \text { Sensors. } & 19, \quad 861 \quad \text { (2019). }\end{array}$ https://doi.org/10.3390/s19040861

32. Ye, Y., Huang, P., Sun, Y., Shi, D.: MBSNet: A deep learning model for multibody dynamics simulation and its application to a vehicle-track system. Mechanical Systems and Signal Processing. 157, 107716 (2021). 
https://doi.org/10.1016/j.ymssp.2021.107716

33. Sun, R.-B., Yang, Z.-B., Yang, L.-D., Qiao, B.-J., Chen, X.-F., Gryllias, K.: Planetary gearbox spectral modeling based on the hybrid method of dynamics and LSTM. Mechanical Systems and Signal Processing. 138, 106611 https://doi.org/10.1016/j.ymssp.2019.106611

34. You, D., Shen, X., Liu, G., Wang, G.: Signal anomaly identification strategy based on Bayesian inference for nuclear power machinery. Mechanical Systems and Signal Processing. 161, $107967 \quad$ (2021). https://doi.org/10.1016/j.ymssp.2021.107967

35. Zhang, L., Ding, H., Shi, J., Huang, Y., Chen, H., Guo, K., Li, Q.: An Adaptive Backstepping Sliding Mode Controller to Improve Vehicle Maneuverability and Stability via Torque Vectoring Control. IEEE Trans. Veh. Technol. 69, 2598-2612 (2020). https://doi.org/10.1109/TVT.2019.2950219

36. Zhang, W., Wang, Z., Drugge, L., Nybacka, M.: Evaluating Model Predictive Path Following and Yaw Stability Controllers for Over-Actuated Autonomous Electric Vehicles. IEEE Trans. Veh. Technol. 69, 12807-12821

(2020). https://doi.org/10.1109/TVT.2020.3030863

37. Donghyun Kim, Sungho Hwang, Hyunsoo Kim: Vehicle Stability Enhancement of Four-Wheel-Drive Hybrid Electric Vehicle Using Rear Motor Control. IEEE Trans. Veh. Technol. 57, 727-735 (2008). https://doi.org/10.1109/TVT.2007.907016

38. Novara, C., Ruiz, F., Milanese, M.: Direct Identification of Optimal SM-LPV Filters and Application to Vehicle Yaw Rate Estimation. IEEE Trans. Contr. Syst. Technol. 19, 5-17 (2011). https://doi.org/10.1109/TCST.2010.2070505

39. Sun, K., Lee, S.T., Zhang, P.: An Adaptive Power System Equivalent for Real-Time Estimation of Stability Margin Using Phase-Plane Trajectories. IEEE Trans. Power Syst. 26, 915-923 (2011). https://doi.org/10.1109/TPWRS.2010.2055900

40. Zheng, Y., Zhou, J., Zhu, W., Zhang, C., Li, C., Fu, W.: Design of a multi-mode intelligent model predictive control strategy for hydroelectric generating unit. Neurocomputing. 207, 287-299 (2016). https://doi.org/10.1016/j.neucom.2016.05.007 